\title{
Somatic Symptoms as Mediating Factors between the Perceived Social Support and the Health-Related Quality of Life in North Korean Defectors
}

\author{
Sung-Doo Won ${ }^{1}$, So Hee Lee ${ }^{\otimes}$, SeogJu Kim ${ }^{3}$, Jin Yong Jun ${ }^{4}$, and Sang-Soo Shin ${ }^{5}$ \\ ${ }^{1}$ Department of Clinical Psychology, Keyo Hospital, Uiwang, Republic of Korea \\ 2Department of Psychiatry, National Medical Center, Seoul, Republic of Korea \\ ${ }^{3}$ Department of Psychiatry, Samsung Medical Center, School of Medicine Sungkyunkwan University, Seoul, Republic of Korea \\ ${ }^{4}$ Department of Social Psychiatry and Rehabilitation, National Center for Mental Health, Seoul, Republic of Korea \\ ${ }^{5}$ Institute for Health and Society, Hanyang University, Seoul, Republic of Korea
}

Objective This study was conducted to examine the mediating effect of somatic symptoms between perceived social support and health-related quality of life among North Korean defectors living in South Korea.

Methods A total of 200 North Korean defectors were assessed using the Multidimensional Scale of Perceived Social Support, the Short-Form 8-Item Health Survey, the Patient Health Questionnaire 15, the PTSD Checklist Civilian Version and the Patient Health Questionnaire 9.

Results Somatic symptoms accounted for physical health-related quality of life after controlling for PTSD and depressive symptoms, but did not for mental health-related quality of life. Moreover, somatic symptoms mediated the relationship between perceived social support and physical and mental health-related quality of life, indicating that perceived social support indirectly affects physical and mental health-related quality of life through somatic symptoms in North Korean defectors.

Conclusion The results of this study suggest that not only intervention for PTSD or depressive symptoms but also for somatic symptoms might be effective to improve physical and mental health-related quality of life for North Korean defectors, especially those who have a lack of perceived social support.

Psychiatry Investig 2017;14(4):407-412

Key Words Perceived social support, Health-related quality of life, Somatic symptoms, North Korean defectors, Mediating effects.

\section{INTRODUCTION}

According to the formal report ${ }^{1}$ from government, 28,795 defectors are tallied on December 2015, a little attention to their mental health has heretofore been received. As far as their quality of life is concerned, which is known as the critical component for their adaptation in South Korea, ${ }^{2}$ the Health-related Quality of Life (HR-QoL) might be one of the major markers for prevention of a variety of psychiatric disorders, such as depression and anxiety. ${ }^{3}$

Received: February 2, 2016 Revised: July 2, 2016

Accepted: August 3, 2016 Available online: May 16, 2017

$\triangle$ Correspondence: So Hee Lee, MD, PhD

Department of Psychiatry, National Medical Center, 245 Eulchi-ro, Jung-gu, Seoul 04564, Republic of Korea

Tel: +82-2-2260-7311, Fax: +82-2-2268-5028, E-mail: psyhee@hanmail.net

(a) This is an Open Access article distributed under the terms of the Creative Commons Attribution Non-Commercial License (http://creativecommons.org/licenses/bync/4.0) which permits unrestricted non-commercial use, distribution, and reproduction in any medium, provided the original work is properly cited.
It is reported that the North Korean defectors experienced various psychological problems before and during defecting to South Korea, as well as after the adaptation. Common mental disorders observed among the defectors were Posttraumatic stress disorder (PTSD), anxiety disorder, and depressive disorders. ${ }^{4}$ In addition to the high prevalence rates of these mental problems, they suffered from somatic symptoms, such as breathing difficulty, palpitation and pseudoneurological symptoms. ${ }^{5}$ With respect to the somatic symptoms, recent studies have revealed the importance of the somatic symptoms in accounting for the association between physical and mental HR-QoL. ${ }^{6}$ It is because that the somatic symptoms are considered additional avenues that might be provided for clinical intervention.

Another significant factor is the social support, which is one of the well-documented predictors that influence on QoL. Previous studies demonstrated the close association between social support and QoL using different samples, such 
as patients with HIV/AIDS or obesity. ${ }^{7,8}$ As far as North Korean defectors are concerned, they are likely to receive poor social support due to the fact that they do not share similar social backgrounds with South Koreans.,10

Several publications have provided how social support is associated with PTSD. Specifically, their social support is negatively associated with PTSD ${ }^{11}$ and depression. ${ }^{12}$ With respect to the depression and anxiety symptoms, negative correlation was found in the adolescent samples from North Korean defectors. ${ }^{13}$ Furthermore, the social support as being a pathway for physical health through psychological, behavioral, and physiological process has been taken into account. ${ }^{10}$

These previous studies in large part provide significant insight into more systematic research. However, what is not seriously considered is the distinction between the perceived social support and the received social support. The received social support is an actual support received given by others in various interpersonal relations, while the perceived social support is of an individual's belief that he/she may get support from others anytime when it is needed. ${ }^{14}$ It seems that there is a consensus that the recipient's perception is more important than the actual provision or presence as social support on health and adaptation. ${ }^{15}$ Consequently, no research examined the association between perceived social support and HR-QoL of the North Korean defectors. Moreover, the pathway of the perceived social support on the HR-QoL is not clearly known.

Thus, the aim of this study is to determine contributing factors, including PTSD, depression, somatic symptoms, and perceived social support on the HR-QoL of North Korean defectors. In addition, we will suggest a model that describes how these factors influence on the HR-QoL.

\section{METHODS}

A total of 200 subjects aged 19 to 76 years were recruited from health call centers and counseling centers caring for North Korean defectors. Those who were considered having intellectual problems or physical disabilities were excluded in this study. All participants provided a written consent andvoluntarily took part in the survey. The subjects were asked to complete the self-administered questionnaires. When the self-administered questionnaires were difficult for them to comprehend, additional explanations were provided by researchers. The study was approved by the National Medical Center's Institutional Review Board.

\section{Patient Health Questionnaire 15}

The Patient Health Questionnaire 15 (PHQ-15) ${ }^{16}$ was used in order to measure 15 somatic symptoms, such as stomach pain, back pain, headache, dizziness, and nausea. Subjects were asked to rate the severity of 15 symptoms as 0 ("not bothered at all"), 1 ("bothered a little"), or 2 ("bothered a lot") for the last 4 weeks. Thus, total scores of the PHQ-15 range from 0 to 30 . Scores of $\geq 5, \geq 10, \geq 15$ represent different levels of somatic symptoms, each of which indicates mild, moderate, and severe levels of the symptoms, respectively. The PHQ-15 has been translated and validated in Korean. ${ }^{17}$ The Korean version of the PHQ-15 has shown good validity and reliability.

\section{Patient Health Questionnaire 9}

Depressive symptoms were assessed with the Patient Health Questionnaire 9 (PHQ-9) ${ }^{18}$ in order to identify severity of the symptoms. Each of the nine PHQ-9 items corresponds to the Diagnostic and Statistical Manual of Mental Disorders Fourth Edition (DSM-IV) diagnostic criteria that measure the major depressive disorder. Subjects were asked how often had experienced the depressive symptoms over the last two weeks. The subjects rated the severity of 9 symptoms as "not at all", "several days", "more than half the days", or "nearly every day", and scored as 0, 1, 2 and 3, respectively. The total scores of PHQ-9 range from 0 to 27. The PHQ-9 has also been translated and validated in Korean. ${ }^{19}$ The Korean version of the PHQ-9 has shown good validity and reliability. A score of 10 is suggested as the optimal cutoff point when screening for depressive disorders using the PHQ-9.

\section{PTSD Checklist civilian version}

The PTSD Checklist civilian version (PCL-C), which corresponds to DSM-IV, consists of 17 items and assesses PTSD symptoms. ${ }^{20}$ A 5-point scale from 1 ("not at all") to 5 ("extremely") was used to rate how much the specific symptom was a problem to them over the past month. The PCL-C total scores with higher numbers are indicative of more PTSD symptoms. A total score of 44 or more suggests probable diagnosis of PTSD among the civilian population. ${ }^{21}$ The PCL$\mathrm{C}$ has also been translated and validated in Korean. ${ }^{22}$ The Korean version of the PCL-C has shown good validity and reliability.

\section{Multidimensional Scale of Perceived Social Support}

The Multidimensional Scale of Perceived Social Support (MSPSS) was used to assess the perceived social support. ${ }^{23}$ The MSPSS consists of 12 items which cover three different types of relations: Family, Friends and Significant others. In this study, the Korean version of the MSPSS was used. ${ }^{24}$ Items were measured on a 5-point scale from 1 ("strongly disagree") to 5 ("strongly agree"). Items were summed and the higher the score, the higher the perceived social support. 


\section{Medical Outcome Short Form 8-Item Health Survey (SF-8)}

The SF-8 was developed to measure the health related quality of life. ${ }^{25}$ How to score and interpret is as follows. First, eight different sub-areas are identified (e.g., Physical Functioning, Role Limitation due to Physical Problems, Bodily Pain, General Health Perceptions, Vitality, Social Functioning, Role Limitation due to Emotional Problems, and General Mental Health). The eight sub-areas yield two representative summaries of the scores, namely, the physical component summary (PCS) and mental component summary (MCS). Each component represents physical health-related quality of life, and mental health-related quality of life, respectively. The range for the summary scores is 0 to 100 , respectively. The Korean version of the SF- 8 has also been validated. ${ }^{26}$

\section{Statistical analysis}

A statistical analysis was performed using IBM SPSS Statistics version 20.0 (Armonk, NY:IBM Corp; 2011, USA) and IBM SPSS AMOS 20.0 (Arbuckle JL. Chicago: SPSS; 2011, USA). IBM SPSS Statistic version 20.0 was used to descriptive statistics analyses, Pearson's correlation analysis, and hierarchical multiple regression analysis. Pearson's correlation analysis was used to determine the strength of the association among the somatic symptoms, depressive symptoms, PTSD symptoms, perceived social support, and health-related quality of life. Hierarchical multiple regression analysis was used to examine the impact of somatic symptoms on the healthrelated quality of life even after controlling for depressive symptoms and PTSD symptoms. With structural equation modeling using AMOS 20.0, we examine the role of somatic symptoms mediating the relation of perceived social support to health-related quality of life. A p-value of 0.05 or less was considered as statistically significant.

\section{RESULTS}

\section{Descriptive data of subjects}

Descriptive statistics including means (M), standard deviations (SD), and range for the all variables are presented in Table 1. Both the mean PHQ-15 (M=12.63, SD=6.67) and PHQ$9(\mathrm{M}=10.60, \mathrm{SD}=8.33)$ scores were moderately in severe range. However, the mean PCL-C score was below the suggested cut-off score of $44(\mathrm{M}=41.20, \mathrm{SD}=19.06)$. Both the mean PCS $(\mathrm{M}=37.87, \mathrm{SD}=10.10)$ and $\mathrm{MCS}(\mathrm{M}=41.29, \mathrm{SD}=11.99)$ scores were approximately $1 \mathrm{SD}$ below the norm of 50 .

Relationships between somatic symptoms, depressive symptoms, PTSD, perceived social support and HR-QoL

Prior to conducting the hierarchical multiple regression
Table 1. Descriptive statistics for the sample

\begin{tabular}{|c|c|c|}
\hline Variables & $\begin{array}{c}\text { Mean (SD) or } \\
\text { number }(\%)\end{array}$ & Range \\
\hline Age & $47.67(11.12)$ & $19-76$ \\
\hline \multicolumn{3}{|l|}{ Gender } \\
\hline Female & $172(86)$ & \\
\hline Male & $28(14)$ & \\
\hline \multicolumn{3}{|l|}{ South Korea entry year (\%) } \\
\hline $2000-2008$ & 40 & \\
\hline 2009-2013 & 60 & \\
\hline \multicolumn{3}{|l|}{ Staying period in } \\
\hline \multicolumn{3}{|l|}{$3 r d$ country $(\%)$} \\
\hline Under 1 year & 37 & \\
\hline Under 3 years & 10 & \\
\hline Under 6 years & 18 & \\
\hline 6 years or more & 35 & \\
\hline Yearly income (10000 KRW) & $1252.97(1120.79)$ & $0-6000$ \\
\hline PHQ-15 & $12.63(6.67)$ & $0-30$ \\
\hline PHQ-9 & $10.60(8.33)$ & $0-27$ \\
\hline PCL-C & $41.20(19.06)$ & $17-85$ \\
\hline MSPSS & $41.33(12.18)$ & $12-60$ \\
\hline PCS & $37.87(10.10)$ & $13.40-61.30$ \\
\hline MCS & 41.29 (11.99) & $15.00-61.40$ \\
\hline
\end{tabular}

N's range from 100 to 200 due to occasional missing data. PHQ15: Patient Health Questionnaire 15, PHQ-9: Patient Health Questionnaire 9, PCL-C: PTSD Checklist civilian version, MSPSS: Multidimensional Scale of Perceived Social Support, PCS: Medical Outcome Short Form 8-Item Health Survey Physical Component Summary, MCS: Medical Outcome Short Form 8-Item Health Survey Mental Component Summary

analyses, correlation analyses were performed to examine the relationships between PHQ-15, PHQ-9, PCL-C, MSPSS, PCS, and MCS. The results of these analyses can be found in Table 2. All of the correlation coefficients were statistically significant. Especially, the PHQ-15 scores were positively correlated with the PHQ- 9 and PCL-C, $r=0.69,0.71$, respectively.

\section{Effects of somatic symptoms on the HR-QoL}

In the first hierarchical multiple regression analysis, depressive symptoms accounted for $25 \%$ of physical health-related quality of life, but PTSD symptoms did not. Somatic symptoms accounted for $8 \%$ of physical health-related quality of life, even when controlling for depressive symptoms and PTSD symptoms (Table 3). In the second analysis, $61 \%$ of the variance in mental health-related quality of life was accounted by depressive symptoms (47\%) and PTSD symptoms (14\%). However, when controlling for depressive symptoms and PTSD symptoms, somatic symptoms did not account for mental health-related quality of life (Table 3). We also checked 
Table 2. Correlations among PHQ-15, PHQ-9, PCL-C, MSPSS, PCS, MCS

\begin{tabular}{lccccc}
\hline \multicolumn{1}{c}{ Variables } & 1 & 2 & 3 & 4 & 5 \\
\hline 1. PHQ-15 & - & & & & \\
2. PHQ-9 & $0.69^{* *}$ & - & & & \\
3. PCL-C & $0.71^{* *}$ & $0.81^{* *}$ & - & & \\
4. MSPSS & $-0.36^{* *}$ & $-0.50^{* *}$ & $-0.46^{* *}$ & - & - \\
5. PCS & $-0.55^{* *}$ & $-0.50^{* *}$ & $-0.44^{* *}$ & $0.24^{* *}$ & $0.30^{* *}$ \\
6. MCS & $-0.56^{* *}$ & $-0.69^{* *}$ & $-0.78^{* *}$ & $0.36^{* *}$ & - \\
\hline
\end{tabular}

${ }^{* *} \mathrm{p}<0.01$. PHQ-15: Patient Health Questionnaire 15, PHQ-9: Patient Health Questionnaire 9, PCL-C: PTSD Checklist civilian version, MSPSS: Multidimensional Scale of Perceived Social Support, PCS: Medical Outcome Short Form 8-Item Health Survey Physical Component Summary, MCS: Medical Outcome Short Form 8-Item Health Survey Mental Component Summary

Table 3. Influence of depressive, PTSD, and somatic symptom severity on physical and mental health-related quality of life

\begin{tabular}{|c|c|c|c|c|}
\hline Models & $\mathrm{R}^{2}$ & $\mathrm{R}^{2}$ change & F change & $\mathrm{p}$ \\
\hline \multicolumn{5}{|l|}{ Model 1 (PCS=dependent variable) } \\
\hline Depressive symptom severity & 0.25 & 0.25 & 65.87 & $<0.001$ \\
\hline Depressive symptom severity and PTSD symptom severity & 0.25 & 0.00 & 0.88 & 0.35 \\
\hline Depressive symptom severity, PTSD symptom severity, and somatic symptom severity & 0.33 & 0.08 & 23.25 & $<0.001$ \\
\hline \multicolumn{5}{|l|}{ Model 2 (MCS=dependent variable $)$} \\
\hline Depressive symptom severity & 0.47 & 0.47 & 177.46 & $<0.001$ \\
\hline Depressive symptom severity and PTSD symptom severity & 0.61 & 0.14 & 72.18 & $<0.001$ \\
\hline Depressive symptom severity, PTSD symptom severity, and somatic symptom severity & 0.61 & 0.00 & 0.17 & 0.68 \\
\hline
\end{tabular}

PCS: Medical Outcome Short Form 8-Item Health Survey Physical Component Summary, MCS: Medical Outcome Short Form 8-Item Health Survey Mental Component Summary

for multicollinearity between depressive symptoms, PTSD symptoms, and somatic symptoms. There was no evidence of multicollinearity among these variables (Tolerance 0.31-0.46, VIF 2.18-3.38).

\section{Path model for the mediating effects of somatic symptoms between perceived social support and physical and mental health-related quality of life}

The hypothetical model suggests that somatic symptoms mediate the relation between perceived social support, physical and mental health-related quality of life, and that perceived social support directly affects physical and mental health-related quality of life. In contrast, the competitive model suggests that somatic symptoms fully mediate the relation between perceived social support, physical and mental healthrelated quality of life, and that perceived social support did not has direct effect on physical and mental health-related quality of life. To examine model fit, comparative fit index (CFI), Tucker-Lewis index (TLI), and root mean square error of the approximation (RMSEA) were evaluated. The overall model fit was good for both models (hypothetical model: CFI=0.98, TLI=0.96, RMSEA=0.09; competitive model: $\mathrm{CFI}=0.97$, TLI=0.95, RMSEA $=0.10$ ). Thus, to determine more appropriate models, $\chi^{2}$ difference test was performed. It indicated that the hypothetical model was superior to the

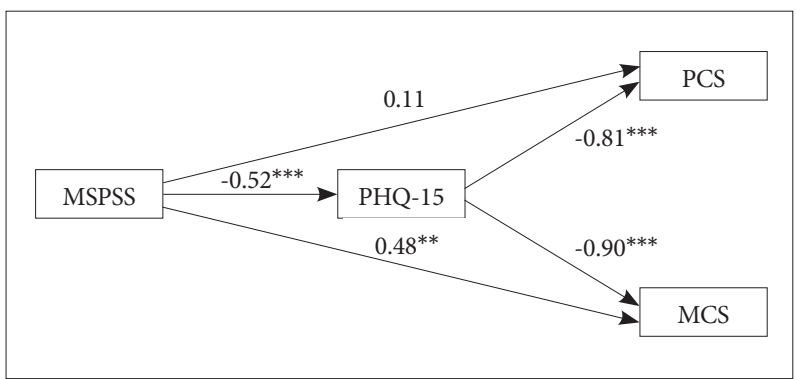

Figure 1. Final model of the relationship between Perceived social support, Somatic symptoms severity, Physical and mental health-related quality of life. ${ }^{* *} p<0.01,{ }^{* * *} p<0.001$. MSPSS: Multidimensional Scale of Perceived Social Support, PHQ-15: Patient Health Questionnaire 15, PCS: Medical Outcome Short Form 8-Item Health Survey Physical Component Summary, MCS: Medical Outcome Short Form 8-Item Health Survey Mental Component Summary.

competitive model $\left(\chi^{2}\right.$ difference $\left.=7.94, \mathrm{df}=2, \mathrm{p}<0.05\right)$. Ultimately, we decided to use the hypothetical model as the final model (Figure 1). Estimated parameters in the final model can be found in Table 4. In the final model, perceived social support accounted for $11 \%$ of somatic symptoms. Perceived social support and somatic symptoms explained $31 \%$ of the variance in physical health-related quality of life, and $34 \%$ of the variance in mental health-related quality of life. Finally, to evaluate the significance of the indirect effects, we performed bootstrap procedure. ${ }^{27}$ The results show that somatic 
Table 4. Parameters of final model

\begin{tabular}{lccccc}
\hline \multicolumn{1}{c}{ Path } & B & SE & $\beta$ & $\mathrm{t}$ & $\mathrm{p}$ \\
\hline Perceived social support $\rightarrow$ Somatic symptom severity & -0.52 & 0.11 & -0.34 & -4.82 & $<0.001$ \\
Somatic Symptoms $\rightarrow$ Physical HR-QoL & -0.81 & 0.10 & -0.53 & -8.48 & $<0.001$ \\
Somatic Symptoms $\rightarrow$ Mental HR-QoL & -0.90 & 0.11 & -0.50 & -8.01 & $<0.001$ \\
Perceived social support $\rightarrow$ Physical HR-QoL & 0.11 & 0.15 & 0.05 & 0.75 & 0.46 \\
Perceived social support $\rightarrow$ Mental HR-QoL & 0.48 & 0.18 & 0.17 & 2.72 & 0.007 \\
\hline
\end{tabular}

Physical HR-QoL: Physical health-related quality of life, Mental HR-QoL: Mental health-related quality of life

symptoms have a statistically significant mediating effect $(95 \%$ $\mathrm{CI}=0.22-0.67$ for physical health-related quality of life and $95 \% \mathrm{CI}=0.24-0.74$ for mental health-related quality of life).

\section{DISCUSSION}

The current study examined the associations among the several variables that influence on the HR-QoL in North Korean defectors. We suggested a model for the mediation effect of somatic symptoms on the relation between social support and HR-QoL. The followings are the detailed results we have found.

First, depressive symptoms, PTSD severity, and somatic symptoms were positively correlated with each other among North Korean defectors. It is consistent with previous studies ${ }^{28,29}$ demonstrating the positive associations between PTSD severity and somatic symptoms. Indeed, a study found that the rate of somatic illness was $81.8 \%$ in the samples of the North Korean defectors. ${ }^{2}$ The present study also found that depressive symptoms and somatization are likely to affect each other as a risk factor. ${ }^{30}$

Second, we were able to expect that the severity of the somatic symptoms can account for physical HR-QoL after controlling for PTSD and depressive symptoms, which are consistent with a previous study's findings. ${ }^{6}$ Contrary to our expectation, the somatic symptoms were not identified as factors that accounted for mental health HR-QoL after controlling for PTSD and depressive symptoms. In fact, a study also found that the incremental accounts of somatic symptoms was only 3.38\% on mental HR-QoL after controlling for PTSD and depressive symptoms, while $17.7 \%$ on physical HR-QoL. ${ }^{6}$ Thus, it suggests that somatic symptoms may have a differential effect on physical as well as mental HR-QoL.

Third, we confirmed that somatic symptoms mediated the relation between the perceived social support and the QoL, suggesting that lower perceived social support might increase somatic symptoms resulting in decreased physical and mental HR-QoL. However, perceived social support had not a direct effect on the physical HR-QoL while had a significant direct effect on the mental health HR-QoL. This result is inconsistent with the previous study ${ }^{31}$ that perceived social support had a direct effect on pain severity, one of the subcomponents of the physical HR-QoL. However, the finding of the present study is consistent with which didn't predict the physical HR-QoL in patients with acute myocardial infarction. ${ }^{32}$ Given this results, there might be a variety of another pathway in the association between the perceived social support and the physical HR-QoL.

Finally, these results suggest that there is a need to concern for the quality life of North Korean defectors by means of improving social support, and clinical interventions for the somatic symptoms, depression, and anxiety. In particular, we should attempt to develop and provide a somatic symptomsfocused intervention to improve mental health among North Korean defectors. Specifically, an intervention needs to include a cognitive restructuring as well as psychoeducation for somatic symptoms.

This study has several limitations that should be noted. First, due to the lack of sample representativeness, $86 \%$ of total participants were women. Thus, the present study may not be generalized to all the North Korean defectors have equal status of affairs. Second, we used only self-report methods to measure the variables of interest, which might lead to either underestimate or overestimate psychiatric symptoms. Moreover, we did not consider another issue, such as cultural and linguistic background of North Koreans, except for the PCL$\mathrm{C}$ assessing PTSD. ${ }^{22}$ Third, when testing the structural equation modeling with several intervening variables, one cannot determine which pathway works more significant. Because of this problem, we did not include depression and PTSD severity in the structural equation models, but examined the factors that influence on the severity of HR-QoL using hierarchical regression analysis. Finally, although the causal relationship among related variables was confirmed, we could consider the hypothesis that defectors with poor physical and mental health have more somatic symptoms, and because of the distress from somatic symptom, they perceived less social support. Consequently, a future study which is more comprehensive could enlighten the temporal sequence of the related-variables.

In sum, this study demonstrated that the associations among PTSD, depressive symptoms, somatization, social support, and 
quality of life in North Korean defectors. It also found that somatization tendency mediated the relation between social support and quality of life. Accordingly, there is a need to pay attention to somatic symptoms and develop alternatives to improve mental health among North Korean defectors. Specifically, an intervention for increasing social support can results in reduction of somatic symptom, and then enable better the quality of life in North Korean defectors.

\section{Acknowledgments}

This research was supported by Research Program funded by National Medical Center, Research Institute (NMC2013PR-03).

\section{REFERENCES}

1. Ministry of Unification. Statistics-North Korean Refugee Policy. Available at: http://www.unikorea.go.kr/content.do? cmsid=3099. Accessed Jan 12, 2016.

2. Han NY, Lee SH, Yoo SY, Kim SJ, Jun JY, Won SD, et al. Predictors of PTSD among North Korean defectors visited psychiatric department of North Korean defectors treatment center. J Korean Neuropsychiatr Assoc 2015;54:105-111.

3. Kim YH, Jeon WT, Cho YA. A study on the prevalence and the influencing factors of the mental health problems among recent migrant North Koreans: a focus on 2007 entrants. Unification Policy Stud 2010; 19:141-174

4. Kim BC. North Korean's mental health. Ment Health Policy Forum 2010;4:19-37.

5. Ahn EM, Song JI, Kang HS, Park JJ, Yoo SH, Huh BR. The symptom expression and the illness behavior of North Korean defectors: toward an effective therapeutic relationships. Korean J Fam Med 2007;28:352358.

6. Cyr KS, McIntyre-Smith A, Contractor AA, Elhai JD, Richardson JD. Somatic symptoms and health-related quality of life among treatmentseeking Canadian Forces personnel with PTSD. Psychiatry Res 2014; 218:148-152.

7. Bekele T, Rourke SB, Tucker R, Greene S, Sobota M, Koornstra J, et al. Direct and indirect effects of perceived social support on health-related quality of life in persons living with HIV/AIDS. AIDS Care 2013;25: 337-346.

8. Herzer M, Zeller MH, Rausch JR, Modi AC. Perceived social support and its association with obesity-specific health-related quality of life. J Dev Behav Pediatr 2011;32:188-195.

9. Turner RJ, Frankel BG, Levin DM. Social support: conceptualization, measurement, and implications for mental health. Res Community Ment Health 1983;3:67-111.

10. Uchino BN. Social support and health: a review of physiological processes potentially underlying links to disease outcomes. J Behav Med 2006;29:377-387.

11. Brewin CR, Andrews B, Valentine JD. Meta-analysis of risk factors for posttraumatic stress disorder in trauma-exposed adults. J Consult Clin Psychol 2000;68:748-766.

12. Kim MR. The adjustment stress and the effect of the social support on the adaptation of the North Korean defectors. Korean Acad Soc Welfare 2004;10:540-565.

13. Park YS. A study on the characteristics of social support and social adjustment of North Korean refugee adolescents in South Korea. Forum Youth Culture 2008;19:79-117.

14. Norris FH, Kaniasty K. Received and perceived social support in times of stress: a test of the social support deterioration deterrence model. J Pers Soc Psychol 1996;71:498-511.

15. Hogan BE, Linden W, Najarian B. Social support interventions: do they work? Clin Psychol Rev 2002;22:383-442.

16. Kroenke K, Spitzer RL, Williams JB. The PHQ-15: validity of a new measure for evaluating the severity of somatic symptoms. Psychosom Med 2002;64:258-266.

17. Han C, Pae CU, Patkar AA, Masand PS, Kim KW, Joe SH, et al. Psychometric properties of the Patient Health Questionnaire-15 (PHQ-15) for measuring the somatic symptoms of psychiatric outpatients. Psychosomatics 2009;50:580-585.

18. Kroenke K, Spitzer RL, Williams JB. The PHQ-9: validity of a brief depression severity measure. J Gen Intern Med 2001;16:606-613.

19. Choi HS, Choi JH, Park KH, Joo KJ, Ga H, Ko HJ, et al. Standardization of the Korean version of Patient Health Questionnaire- 9 as a screening instrument for major depressive disorder. J Korean Acad Fam Med 2007;28:114-119.

20. Ruggiero KJ, Del Ben K, Scotti JR, Rabalais AE. Psychometric properties of the PTSD Checklist-Civilian Version. J Trauma Stress 2003;16: 495-502.

21. Blanchard EB, Jones-Alexander J, Buckley TC, Forneris CA. Psychometric properties of the PTSD Checklist (PCL). Behav Res Ther 1996; 34:669-673.

22. Oh SI, Won SD, Lee SH, Yoo SY, Kim HC, Kim HJ. Reliability and validity of the Korean version of the PTSD checklist civilian version in North Korean defectors. J Korean Neuropsychiatr Assoc 2014;53:410-417.

23. Zimet GD, Dahlem NW, Zimet SG, Farley GK. The multidimensional scale of perceived social support. J Pers Assess 1988;52:30-41.

24. Kang MO. The benefits of social support in the relationship between adolescent group bullying and emotional stability of the victims. Changwon, South Korea: Kyungnam University; 2011.

25. Ware JE, Kosinski M, Dewey JE, Gandek B. How to Score and Interpret Single-item Health Status Measures: A Manual for Users of the of the SF-8 Health Survey. Lincoln: QualityMetric, Incorporated; 2001.

26. Han C, Lee E, Iwaya T, Kataoka H, Kohzuki M. Development of the Korean version of Short-Form 36-Item Health Survey: health related QOL of healthy elderly people and elderly patients in Korea. Tohoku J Exp Med 2004;203:189-194.

27. Shrout PE, Bolger N. Mediation in experimental and nonexperimental studies: new procedures and recommendations. Psychol Methods 2002;7:422-445.

28. Osorio C, Carvalho C, Fertout M, Maia A. Prevalence of post-traumatic stress disorder and physical health complaints among Portuguese Army Special Operations Forces deployed in Afghanistan. Mil Med 2012;177:957-962.

29. Pacella ML, Hruska B, Delahanty DL. The physical health consequences of PTSD and PTSD symptoms: a meta-analytic review. J Anxiety Disord 2013;27:33-46.

30. van Ravenzwaaij J, Olde Hartman T, van Ravesteijn H, Eveleigh R, van Rijswijk E, Lucassen P. Explanatory models of medically unexplained symptoms: a qualitative analysis of the literature. Ment Health Fam Med 2010; 7:223-231.

31. Lackner JM, Brasel AM, Quigley BM, Keefer L, Krasner SS, Powell C, et al. The ties that bind: perceived social support, stress, and IBS in severely affected patients. Neurogastroenterol Motil 2010;22:893-900.

32. Bucholz EM, Strait KM, Dreyer RP, Geda M, Spatz ES, Bueno H, et al. Effect of low perceived social support on health outcomes in young patients with acute myocardial infarction: results from the VIRGO (Variation in Recovery: Role of Gender on Outcomes of Young AMI Patients) study. J Am Heart Assoc 2014;3:e001252. 